\title{
Avaliação das características poliméricas do biofilme do resíduo de melancia
}

Evaluation of the polymeric characteristics of the biofilm of the agricultural residue of watermelon

\author{
A. C. P. de Menezes Filho*; J. C. P. de Souza; C. F. de S. Castro \\ Agroquímica/Laboratório de Química Tecnológica, Instituto Federal de Educação, Ciência e Tecnologia Goiano, \\ Campus Rio Verde, 75901-970, Rio Verde-Goiás, Brasil
}

*astronomoamadorgoias@gmail.com

(Recebido em 20 de julho de 2019; aceito em 19 de agosto de 2019)

\begin{abstract}
O trabalho teve por objetivo avaliar as características poliméricas do biofilme a partir do resíduo do fruto de Citrullus lanatus. O biopolímero foi produzido a partir de uma suspensão aquosa farinácea do albedo e avaliado quanto às características físico-químicas, antioxidantes, mecânicas e por análises em microscopia óptica e eletrônica por varredura. O biofilme do resíduo de melancia apresentou importantes resultados nos testes físico-químicos, mecânicos e de atividade antioxidante, importante quantitativo de fenóis totais e boa eficiência nas características estruturais. Este novo biopolímero a partir do resíduo de melancia poderá ser aplicado no desenvolvimento de embalagens biodegradáveis, bem como no revestimento de salames defumados na indústria alimentícia.
\end{abstract}

Palavras-chave: filme biodegradável, Citrullus lanatus, propriedades mecânicas.

The objective of this work was to evaluate the polymeric characteristics of biofilm from Citrullus lanatus fruit residue. The biopolymer was produced from an albedo farinaceous aqueous suspension, and evaluated for physicochemical, antioxidant, mechanical characteristics and analysis by optical and scanning electron microscopy. The biofilm of watermelon residue showed important results in physicochemical, mechanical and antioxidant activity tests, important quantity of total phenols and good efficiency in structural characteristics. This new biopolymer from watermelon residue could be applied in the development of biodegradable packaging, as well as in the coating of smoked salami in the food industry.

Keywords: biodegradable film, Citrullus lanatus, mechanical property.

\section{INTRODUÇÃO}

As embalagens possuem a função de acondicionar, proteger e manter a integridade de um produto. As primeiras embalagens foram criadas a partir de formulações de produtos do petróleo, sendo assim chamadas de embalagens sintéticas. As embalagens estão presentes nos mais variados tipos de produtos, e após seu uso são descartadas, permanecendo no ambiente por longos períodos, causando sérios danos ao meio ambiente, quando descartadas incorretamente contaminando os mais diversos ecossistemas [1].

Com isso, foi criada a linha verde (ou natural) de produtos como necessidade de se desenvolver novos materiais a partir de fontes biodegradáveis, como forma de minimizar os danos ao meio ambiente, optando-se pela utilização de materiais naturais que anteriormente eram tratados como resíduos, sendo descartados de forma incorreta sem o reuso apropriado. Os estudos no desenvolvimento de biofilmes tiveram início a partir de matrizes poliméricas de amido, proteína, lipídios e fibras, que apresentam boa eficiência no arranjo intermolecular formando uma solução polimérica com característica de embalagem biodegradável. Os biopolímeros são abundantes na natureza, sendo facilmente encontradas nas frutas, hortaliças, tubérculos, legumes e em diversos tipos de grãos. Atualmente, vem-se empregando o resíduo agrícola da fruticultura na produção de biofilmes, agregando a perda ocasionada ainda na lavoura durante a colheita, bem como no manejo, transporte e na distribuição nos grandes e pequenos mercados varejistas $[1,2]$.

De acordo com Rocha et al. (2014) [1] e Tang et al. (2003) [3], as matrizes poliméricas naturais apresentam efetiva importância na barreira aos gases $\left(\mathrm{CO}_{2}\right.$ e $\left.\mathrm{O}_{2}\right)$, na elasticidade e resistência à tração; sendo estas características importantes para uma embalagem que visa evitar choques 
mecânicos durante o transporte e liberação de vapores durante o armazenamento. Entretanto, essas matrizes naturais apresentam alta permeabilidade ao vapor de água em função do seu caráter hidrofílico, limitando assim sua aplicação. Em contrapartida, o uso de substâncias plastificantes como o glicerol, potencializa as propriedades destes biomateriais garantindo uma baixa troca de vapores de água, bem como o aumento da flexibilidade e a diminuição do efeito quebradiço.

No Brasil atualmente são cultivadas em torno de 48 cultivares de melancia. A melancia é uma fruta pertencente à família das cucurbitáceas, apresentando diferentes tamanhos, formas e cores variadas tanto na casca como na polpa. Dentre estas variedades a espécie Citrullus lanatus Thumb. Mansf. var. Crimson Sweet Extra se destaca pelo seu tamanho avantajado e espesso albedo (entrecasca). Dentre os fatores genéticos dos cultivares, pluviosidade, solo e por estar em uma área de clima tropical, o Brasil é o quarto maior produtor de melancia do mundo, com uma parcela de $80 \%$ da produção mundial, estando atrás da China, Turquia e Irã [4].

Devido a esta grande área de produção, há também uma volumosa perda de frutos ainda na lavoura, gerando grande quantidade de resíduos que não são devidamente aproveitados. Com isso, este estudo objetivou desenvolver e avaliar as características poliméricas de um biofilme a partir do resíduo agrícola de Citrullus lanatus Thumb. Mansf. var. Crisom Sweet Extra.

\section{MATERIAIS E MÉTODOS}

O biofilme foi produzido conforme descrito por Souza et al. (2012) [5], com adaptações. Uma alíquota de $25 \mathrm{~g}$ de farinha do albedo de melancia foi acrescida com $100 \mathrm{~mL}$ de água destilada, a solução foi aquecida a $40{ }^{\circ} \mathrm{C}$ em banho-maria por 1 hora. Em seguida, a solução foi filtrada em pano fino de nylon e o sobrenadante coletado. O sobrenadante foi aquecido a $60{ }^{\circ} \mathrm{C}$ sob homogeneização em agitador magnético. Durante a agitação, foram acrescidos $0,9 \mathrm{~g}$ de glicerol $(\mathrm{m} / \mathrm{v})$ e $10 \mathrm{~mL}$ de solução aquosa de cloreto de cálcio a $1,5 \%(\mathrm{~m} / \mathrm{v})$. Cerca de $25 \mathrm{~mL}$ da solução foi transferida para placa de Petri de polietileno, e em seguida, a placa foi levada para a estufa com circulação de ar forçada a $60{ }^{\circ} \mathrm{C}$ por 3 horas. Após esse tempo, uma segunda alíquota de $25 \mathrm{~mL}$ foi acrescida sobre a primeira camada na placa, formando uma segunda camada filmogênica. Após a adição da segunda camada, a placa ficou sob aquecimento a $60{ }^{\circ} \mathrm{C}$ por 12 horas na estufa com circulação de ar forçada. Após o período de secagem, o biofilme foi retirado da placa de Petri e armazenado em dessecadora com sílica gel até análises.

A espessura do biofilme foi determinada conforme descrito por Rocha et al. (2014) [1], adaptado. Foi utilizado um micrômetro digital (Mitutoyo, Mod. 203-821), com sensibilidade de ( \pm $0,001 \mathrm{~mm}$ ). Cerca de 10 pontos aleatórios no biofilme foram avaliados.

Para determinação dos teores de umidade e cinzas do biofilme, foi utilizada metodologia descrita por IAL (2008) [6] e Abdollahi et al. (2012) [7] com adaptações. Para o teor de umidade (Tu\%), 1 $\mathrm{g}$ de biofilme teve a massa aferida em cadinho previamente seco em forno mufla a $550{ }^{\circ} \mathrm{C}$ por 4 horas. Em seguida, a amostra foi levada para estufa com circulação de ar forçada a $105{ }^{\circ} \mathrm{C}$ por 3 horas. Logo após, o cadinho com amostra foi resfriado e a massa determinada. Para o teor de cinzas (Tcz\%). Após a determinação de umidade, o cadinho com amostra foi levado para forno mufla a $550{ }^{\circ} \mathrm{C}$ por $3 \mathrm{~h}$. Em seguida, o cadinho foi resfriado e sua massa determinada. A diferença de massa entre as amostras foram determinadas, e os resultados expressos em percentagem.

A opacidade do biofilme foi determinada em espectrofotometro UV-Vis (Bell Photonics, Mod. UV-M51), conforme descrito por Rocha et al. (2014) [1]. O biofilme foi cortado e aderido à parede externa de uma cubeta de quartzo com campo único. Como branco instrumental, foi utilizada a mesma cubeta de quartzo sem biofilme. O comprimento de ondas utilizado foi $500 \mathrm{~nm}$. A opacidade foi determinada conforme a equação, $\mathrm{Abs}_{500} / \mathrm{T}$. Onde $\mathrm{Abs}_{500}$ é o resultado em $500 \mathrm{~nm}$ e $\mathrm{T}$ a espessura média do biofilme em $\mathrm{mm}$.

O teste de solubilidade seguiu conforme proposto por Jahed et al. (2017) [8]. O biofilme foi cortado em formato quadrado com $\left(1,5 \mathrm{~cm}^{2}\right)$, e seco em estufa a $105{ }^{\circ} \mathrm{C}$ por $2 \mathrm{~h}$. Em seguida foi determinada a massa. Logo após, foi transferido para um béquer contendo $50 \mathrm{~mL}$ de água ultra pura (Milli-Q, Mod. Advantage A10). O béquer com amostra foi agitado em mesa agitadora orbital (Solab, Mod. SL180/A) sob lenta agitação por $24 \mathrm{~h}$. Após este tempo, a amostra foi removida e seca em estufa a $105^{\circ} \mathrm{C}$ por $6 \mathrm{~h}$. A solubilidade em água foi calculada em percentagem conforme 
equação, \% MS $=\left(M_{i}-M_{f} / M_{i}\right)$ x 100 . Onde, \%MS é a percentagem de material seco solubilizado; $M i$ massa inicial e $M f$ massa final do material seco não solubilizado.

A taxa de inchamento foi determinada conforme descrito por Zhang et al. (2018) [9], com adaptações. Uma amostra de biofilme com $2 \times 2 \mathrm{~cm}$, foi cortado e seco em estufa a $105{ }^{\circ} \mathrm{C}$ por 2 horas. Em seguida, a amostra teve sua massa aferida $\left(M_{i}\right)$. Logo após, foi imersa em $30 \mathrm{~mL}$ de água ultra pura (Milli-Q, Mod. Advantage A10), por 30 min. Após este período, a amostra teve sua massa novamente aferida $\left(M_{f}\right)$ e a taxa de inchamento calculada conforme equação, \% TI $=\left(M_{f}-M_{i} / M_{i}\right) \mathrm{x}$ 100.

O pH foi determinado conforme descrito por Menezes Filho et al. (2019) [10], com adaptações. Uma alíquota de $0,100 \mathrm{~g}$ de biofilme foi diluída em $20 \mathrm{~mL}$ de água destilada e deixada em descanso por $15 \mathrm{~min}$. Em seguida, foi realizado a leitura em pHmetro digital de bancada (Luca, Mod. 210Z). A atividade de água (aw) foi determinada em equipamento HygroPalm (HygroPalm Rotronic, Mod. HP23-AW-A), com uma alíquota de 0,250 g de biofilme, conforme descrito por Menezes Filho et al. (2019) [10] adaptado.

A degradabilidade do biofilme seguiu conforme descrito por Martucci e Ruseckaite (2009) [11], com adaptações. $\mathrm{O}$ experimento foi realizado em formas de polietileno de alta densidade - PEAD, contendo solo do tipo eutroférrico com a flora microbiana natural. A amostra de biofilme foi cortada em $2 \times 3 \mathrm{~cm}$, seguido de secagem em estufa a $60{ }^{\circ} \mathrm{C}$ por 24 horas para remoção da umidade. Em seguida foi resfriado e tomado à massa inicial $\left(M_{i}\right)$. A amostra foi adicionada individualmente em um envelope de polietileno confeccionado. Em seguida o envelope contendo o biofilme foi enterrado a uma profundidade de $6 \mathrm{~cm}$ no solo. A umidade relativa foi mantida em $40 \%$ e com temperatura de $20^{\circ} \mathrm{C}$. A amostra foi removida nos seguintes dias 5, 7, 11 e $15^{\circ}$ dia. Após este período, o filme foi limpo com pincel e seco em estufa a $60{ }^{\circ} \mathrm{C}$ até massa constante $\left(M_{f}\right)$. A biodegradabilidade (\%Bio) foi calculada conforme equação, \%Bio $=\left(M_{f}-M_{i} / M_{i}\right) \times 100$.

A eficácia do biofilme como redutor do radical 2,2-dipicril-hidrazil (DPPH) foi determinada conforme descrito por Jahed et al. (2017) [8], modificado. Uma amostra de $25 \mathrm{mg}$ de filme foi dissolvido em $4 \mathrm{~mL}$ de água destilada. Em seguida, 2,8 mL da solução foi acrescida com $0,2 \mathrm{~mL}$ de uma solução metanólica de DPPH a 0,6 mM. A solução foi agitada e deixada em descanso por $1 \mathrm{~h}$ em local escuro. A leitura foi realizada em espectrofotômetro UV-Vis (Bell Photonics, Mod. UVM51) no comprimento de ondas em $517 \mathrm{~nm}$. A percentagem de inibição radicalar foi determinada conforme equação, \%redução $=\left(\mathrm{Abs}_{\text {controle }}-\mathrm{Abs}_{\text {amostral }} / \mathrm{Abs}_{\text {controle }}\right) \times 100$.

Para determinação da atividade antioxidante pela redução do ferro (FRAP), foi realizado conforme metodologia descrita por Infante et al. (2013) [12]. Inicialmente foi preparado um extrato com $2 \mathrm{~g}$ de biofilme dissolvidos em $20 \mathrm{~mL}$ de uma solução hidroetanólica (80:20) (v/v). A solução foi submetida à radiação por ultrassom à temperatura de $25{ }^{\circ} \mathrm{C}$ por 20 minutos em banho-deultrassom (Eco-sonics, Mod. Q3.0/40) com frequência US: $40 \mathrm{kHz}$. Sendo em seguida, centrifugado a $2500 \mathrm{rpm}$ por 20 minutos. O sobrenadante foi recolhido e utilizado para a análise. Uma alíquota de $100 \mu \mathrm{L}$ do estrato foi acrescido com $3 \mathrm{~mL}$ do reagente de FRAP, e aquecido a 37 ${ }^{\circ} \mathrm{C}$ em banho-maria por 30 min. A leitura foi realizada em espectrofotômetro UV-Vis (Bell Photonics, Mod. UV-M51) a $595 \mathrm{~nm}$. Os resultados foram expressos em $\mu \mathrm{M} \mathrm{Fe}^{2+} / \mathrm{g} \mathrm{MS}$ (massa seca).

Os compostos fenólicos totais foram determinados conforme descrito por Menezes Filho et al. (2019) [13], com adaptações. Partindo da solução de extrato realizado no teste para atividade antioxidante por FRAP, uma alíquota de $0,5 \mathrm{~mL}$ desta solução, foi acrescida com $8 \mathrm{~mL}$ de água destilada e $0,5 \mathrm{~mL}$ de uma solução aquosa de Folin-Ciocalteau na proporção (1:9). Em seguida, foi acrescida com uma solução aquosa de carbonato de sódio anidro a 7,5\% $(\mathrm{m} / \mathrm{v})$. A reação ocorreu por 60 minutos em local escuro. Em seguida, foi realizada a leitura em espectrofotômetro UV-Vis (PerkinElmer, Mod. UV/VIS/NIR Spectrometer, Lambda 750) no comprimento de ondas a $720 \mathrm{~nm}$. Como branco do instrumento foi utilizada água destilada. A partir de uma curva padrão, foi realizado o cálculo do conteúdo de compostos fenólicos totais, sendo expressos em mg equivalente ao ácido gálico EAG $100 \mathrm{~g}^{-1}$ MS (massa seca).

Para determinação da resistência de tração, foi utilizada máquina universal (Instron, Mod. 3360). Foram cortadas tiras de biofilme com 15 x $120 \mathrm{~mm}$ conforme descrito por Silva et al. (2019) [14]. O sistema foi ajustado com espaçamento entre ganchos de $100 \mathrm{~mm}$. A resistência de tração foi realizada com uma velocidade de $0,21 \mathrm{~mm} / \mathrm{s}$, em uma célula de carga máxima de $650 \mathrm{~N}$. Os 
resultados foram obtidos para tensão máxima, tensão de ruptura, alongamento e para módulo de elasticidade.

A avaliação da permeabilidade ao vapor de água foi determinada conforme descrito por Rocha et al. (2014) [1], com adaptações. Uma amostra de biofilme com $35 \mathrm{~mm}$ de diâmetro, foi fixado em uma célula de vidro, contendo água ultra pura (Milli-Q, Mod. Advantage A10). A célula foi acondicionada em dessecador contendo sílica gel e o sistema mantido a temperatura de $25{ }^{\circ} \mathrm{C}$. A determinação de massa da célula foi realizada em intervalos de 24 h após o início, e o término após 9 dias de análises. O resultado em massa da célula de permeação foi determinado em função do tempo em segundos (s), e uma reta foi obtida por regressão linear, através desta determinação, foi encontrado o coeficiente angular. E calculou-se a taxa de permeabilidade ao vapor de água (TVA) onde: TVA - g/T x A, sendo g/t, o coeficiente angular da reta e A a área de permeação $\left(\mathrm{m}^{2}\right)$. A PVA foi calculada conforme equação, PVA $=($ TVA.$x) / \Delta \mathrm{P}$. Onde $x$ é a espessura média dos filmes $(\mathrm{mm})$ e $\Delta \mathrm{P}$ é a diferença de pressão de vapor do ambiente (dessecadora) $\left(0 \mathrm{kPa} 25^{\circ} \mathrm{C}\right)$ e água ultra pura $\left(3,17 \mathrm{kPa} 25^{\circ} \mathrm{C}\right)$.

A análise por colorimetria foi realizada em colorímetro (ColorFlex, Mod. EZ digital) no sistema HunterLab. Os parâmetros de cor avaliados foram $L^{*}, a^{*}$ e $b^{*}$, conforme descrito por Rocha et al. (2014) [1]. O Chroma $\left(C^{*}\right)$ foi avaliado conforme equação, $C^{*}=\sqrt{ }\left(\mathrm{a}^{*}\right)^{2}+\left(\mathrm{b}^{*}\right)^{2}$ e o Hue-Angle $\left(h^{\circ}\right)$ de acordo com a equação, Arctang $=\tan ^{-1}\left(\mathrm{~b}^{*} / \mathrm{a}^{*}\right)$, sendo avaliados conforme descrito por Clerici et al. (2011) [15].

As imagens da área superficial do biofilme e filme convencional (PVC) foram realizadas por microscopia óptica de alta resolução (MOAR) em microscópio óptico com câmera acoplada (Nikon, Mod. Eclipse TS2R). A modelagem matemática foi realizada através de software ImageJ (versão livre 1.8.0_172). Para análise da superfície de contato do filme biodegradável, foi avaliada também utilizando a microscopia eletrônica de varredura (MEV) em microscópio eletrônico de varredura (Jeol, Mod. JSM - 6610 com EDS), com câmera acoplada (Thermo Scientific, Mod. NSS Spectral Imaging), sendo a superfície do biopolímero revestido com uma fina camada de ouro.

Para análise por espectroscopia no infravermelho, amostras de filme convencional e de biofilme foram submetidas à análise em espectrofotômetro com absorção na região média do infravermelho com transformada de Fourier, equipamento (PerkinElmer, mod. Frontier FT-IR/NIR Spectrometer) no módulo Universal ATR Sampling Accessory, no modo de transmitância (\%T), com 8 acúmulos de varreduras e resolução de $2 \mathrm{~cm}^{-1}$. Os espectrogramas foram analisados através do software Spectrum IR.

A análise estatística consistiu na determinação do desvio padrão a partir da média de uma triplica, aplicada em todos os experimentos, seguido de $( \pm)$ desvio padrão. O software utilizado para os dados estatísticos foi o PAST 3 PAlaeontological STatistics (versão 3.25).

\section{RESULTADOS E DISCUSSÃO}

Na Tabela 1 estão apresentados os resultados da avaliação dos parâmetros morfológicos, físicoquímicos, antioxidantes, dos compostos fenólicos totais e das propriedades mecânicas do biopolímero (biofilme) de melancia.

O biofilme de $C$. lanatus apresentou espessura média de $0,145 \mathrm{~mm}$, com aspecto contínuo e sem fraturas. A uniformidade na mensuração das amostras de filmes biodegradáveis implica positivamente nas propriedades mecânicas e de barreira para vapores de gases. De acordo com Silva et al. (2019) [14], biofilmes que apresentam espessura variada em um mesmo lote, pode comprometer o desempenho funcional da embalagem. Estudo realizado por Silva et al. (2019) [14], os pesquisadores obtiveram espessuras de biofilmes mais espessos, para fécula de mandioca incorporados com cafeína irradiada, onde apresentaram resultados entre 0,1783 a 0,2034 $\mathrm{mm}$. Zhang et al. (2018) [9], avaliaram diferentes tratamentos em biofilmes com média de espessura igual a 0,030 mm. Já Henriques et al. (2008) [2], determinaram a espessura média do filme convencional (PVC) onde apresentou média de $0,021 \mathrm{~mm}$.

O teor de umidade foi de $8,45 \%$, e cinzas de $0,056 \%$ para o biofilme de $C$. lanatus Tabela 1. Costa et al. (2017) [16], encontraram para biofilmes de amido do feijão macáçar, teores de umidade 
entre 14,45 a 26,50\%. Já Dantas et al. (2015) [17], avaliaram diferentes formulações de biofilmes, incorporados com polpas de frutas onde encontraram teor de umidade entre 10,93 a 19,69\%.

Tabela 1: Parâmetros morfológicos, físico-químicos, antioxidantes, fenólicos e propriedades mecânicas do biofilme de melancia.

\begin{tabular}{|c|c|}
\hline Parâmetros & Biofilme \\
\hline Espessura (mm) & $0,145 \pm 0,03$ \\
\hline Umidade (Tu\%) & $8,45 \pm 0,13$ \\
\hline Cinzas $(\mathrm{Tcz} \%)$ & $0,056 \pm 0,02$ \\
\hline Opacidade & 3,057 \\
\hline Solubilidade em água (\%SA) & $81,83 \pm 2,78$ \\
\hline Taxa de inchamento (\% TI) & $114,37 \pm 9,89$ \\
\hline pH & $6,1 \pm 0,01$ \\
\hline Atividade de água (aw) & $0,480 \pm 0,001$ \\
\hline Biodegradabilidade (\%Bio) (dia/\% Tu) & 5-11 dias \\
\hline & $15,91 \% \pm 0,12-27,06 \% \pm 0,09$ \\
\hline Atividade antioxidante (\%DPPH) & $75,55 \pm 2,91$ \\
\hline $\operatorname{FRAP}\left(\mu \mathrm{m} \mathrm{g} \mathrm{Fe} \mathbf{~}^{2+}\right)$ & $121,60 \pm 0,12$ \\
\hline Fenólicos totais (mg EAG $100 \mathrm{~g}^{-1} \mathrm{MS}$ ) & $5,64 \pm 0,11$ \\
\hline Tensão de ruptura (MPa) & $1,38 \pm 0,32$ \\
\hline Alongamento (\%) & $2,26 \pm 0,68$ \\
\hline Módulo de elasticidade (MPa) & $91,09 \pm 33,95$ \\
\hline Força de ruptura (N) & $3,01 \pm 0,05 \mathrm{~N}$ \\
\hline Permeabilidade ao vapor de água (PVA) & $0,225 \pm 0,004 \mathrm{~g} \mathrm{~mm} \mathrm{~h}^{-1} \mathrm{~m}^{-2} \mathrm{kPa}$ \\
\hline
\end{tabular}

Milímetros (mm), teor de umidade (Tu\%), teor de cinzas (Tcz\%), solubilidade em água (\%AS), permeabilidade ao vapor de água (PVA), taxa de inchamento (\%TI), biodegradabilidade (\%Bio), mg de ácido gálico por $100 \mathrm{~g}^{-1}$ matéria seca (mg EAG $\left.100 \mathrm{~g}^{-1} \mathrm{MS}\right)$. Médias em triplicata seguida de $( \pm)$ desvio padrão.

A opacidade do biofilme de $C$. lanatus foi de 3,057. Sendo considerado um filme biodegradável de baixa opacidade, apresentando ser altamente translúcido, sendo uma excelente propriedade visual. Este valor foi similar aos observados por Carissimi et al. (2018) [18], onde encontraram para diferentes composições filmogênicas incorporadas com extratos de microalgas, resultados de opacidade entre 1,13 a 9,47. E para o estudo proposto por Rocha et al. (2014) [1], onde obtiveram opacidade para biofilmes entre 2,16 a 4,88. Os pesquisadores complementam que, quanto mais alto o valor, mais opaco é o biofilme, e com isso, sua aplicabilidade é restrita.

A solubilidade em água foi de 81,83\% para o biofilme de melancia. Já Brito et al. (2019) [19], encontraram para biofilmes de resíduos de frutas e legumes, e pectina, resultados entre 49,00 a $96,00 \%$, estando próximos ao deste estudo. O biofilme demonstrou apresentar média atividade hidrofílica. Com esta característica, o biofilme de melancia não pode ser exposto a teores de umidade acima de $83 \%$ quando incorporados com glicerol. O mesmo foi observado em biofilmes incorporados com glicerol no estudo desenvolvido por Rocha et al. (2014) [1]. Possivelmente trabalhos futuros poderão avaliar a produção de uma solução polimérica enriquecida com quitosana ou outros biopolímeros em conjunto (blendas), para que se possa diminuir a solubilidade e aumentar a exposição aos teores de umidade superiores a $60 \%$.

De acordo com Brito et al. (2019) [19], os polímeros naturais não podem ficar expostos a umidade acima de $60 \%$, para que não ocorra a perda da estabilidade das funções do biopolímero. A taxa de inchamento do biofilme de C. lanatus foi de 114,37\%. Já Zhang et al. (2018) [9], avaliando diferentes tratamentos de biofilme a base de quitosana incorporados com óleo essencial, obtiveram variações de inchamento entre 361,42 a $492,09 \%$. Os pesquisadores complementam que a taxa de inchamento é proporcional à quantidade de água absorvida pela matriz polimérica. E que, biofilmes acrescidos com cloreto de cálcio e amido, possuem maior tendência a absorver grandes quantidades de água, devido às características hidrofílicas, consecutivamente, provocando a desestabilidade da matriz polimérica, ocasionando deformações e perdas da capacidade protetora e mecânica. 
O pH do biofilme de $C$. lanatus foi de 6,1. Estudo realizado por Brito et al. (2019) [19], avaliaram biofilmes a partir de resíduos de frutas, legumes e com incorporação de pectina, onde obtiveram pHs entre 4,0 a 4,22 mais ácidos que o encontrado neste estudo. A atividade de água foi de 0,480 aw para o biofilme de C. lanatus. Dantas et al. (2015) [17], encontraram atividade de água em diferentes biofilmes resultados entre 0,660 a 0,700 aw. Já Santana et al. (2013) [20], avaliaram biofilmes de quitosana com atividade de água entre 0,581 a 0,589 aw. Atividade de água acima de 0,600 aw torna os filmes biodegradáveis propensos ao ataque por microorganismos deterioradores. Com isso o biofilme de melancia desenvolvido neste estudo possui ótima característica de atividade de água, aumentando o tempo da vida de prateleira.

O tempo de biodegradabilidade apresentou inchamento no $5^{\circ}$ dia, com taxa de umidade de $15,91 \%$, e massa final de $62,18 \%$. Sua total degradabilidade foi observada no $11^{\circ}$ dia com taxa de umidade de $27,06 \%$ e com $98,86 \%$ de massa final biodegradada. A formulação do polímero natural de $C$. lanatus acrescido com glicerol absorveu água com eficiência, com isso, a microbiota natural do solo agiu com maior eficácia na biodegradabilidade, diminuindo o tempo de vida do produto no ambiente. Tempo de biodegradabilidade superior, foram observados no estudo desenvolvido por Campagner et al. (2014) [21], onde avaliaram biofilmes a base de amido e lignossilfonatos, onde apresentaram biodegradação entre 5 a 8 semanas. Já Martucci \& Ruseckaite (2009) [11], avaliando biofilmes, observaram inchamento também a partir do $5^{\circ}$ dia com taxa de umidade entre 20 a $40 \%$ e após o $14^{\circ}$ dia, apresentaram difícil análise devido à deterioração avançada. $\mathrm{O}$ tempo de biodegradabilidade é uma importante característica no processo de desenvolvimento de embalagens biodegradáveis, visto que, quanto menor o tempo do produto disponível na sua forma íntegra após uso, menor será a sua influência no volume do conteúdo destinado ao descarte, e com isso menor tempo de biodisponibilidade no ambiente quando comparado a embalagens convencionais sintéticas [1].

O biofilme de $C$. lanatus apresentou importantes atividades antioxidantes avaliadas na redução do radical livre DPPH e para FRAP, com resultados de 75,55\% e $121,60 \mu \mathrm{M} \mathrm{g} \mathrm{Fe}{ }^{2+} \mathrm{MS}$, respectivamente, Tabela 1. Kim et al. (2018) [22], avaliaram diferentes filmes de Sargassum fulvellum incorporados com extrato de Aronia melanocarpa, onde obtiveram redução do radical DPPH entre 2,93 a 32,96\%. O teor de compostos fenólicos totais no biofilme foi de 5,64 mg EAG $100 \mathrm{~g} \mathrm{~g}^{-1}$ MS. O conteúdo total de fenóis está diretamente relacionado com a atividade antioxidante medida pela redução dos radicais livres [23].

A tensão de ruptura foi de $1,38 \mathrm{MPa}$, com alongamento de 2,26\% e módulo de elasticidade de 91,09 MPa para o biofilme de C. lanatus. Silva et al. (2019) [14], encontraram para filmes de fécula de mandioca incorporados com cafeína irradiada, tensão de ruptura entre 0,785 a 1,47 MPa, alongamento entre 3,79 a 4,78\% e módulo de elasticidade entre 191,31 a 214,24 MPa. Já Brito et al. (2019) [19], obtiveram tensão de ruptura entre 1,2 a 2,9 MPa, com alongamento entre 16 a 51\%, e módulo de elasticidade entre 0,03 a 0,16 MPa.

A força de ruptura do biofilme de $C$. lanatus apresentou média de 3,01 N, sendo um bom resultado, visto que, o plastificante incorporado permite melhor adesão e maior força de rompimento. Entretanto, de acordo com Rocha et al. (2014) [1], os pesquisadores afirmam que altos teores de glicerol podem declinar a força de ruptura. Vale ressaltar que biofilmes incorporados com proteínas podem produzir uma força de ruptura menor, não sendo uma boa opção no desenvolvimento de biopolímeros [1]. Silva et al. (2019) [14], obtiveram tensão de ruptura entre 3,5749 a 5,7698 $\mathrm{N}$ para biofilmes de fécula de mandioca incorporados. Zhang et al. (2018) [9], avaliaram biofilmes a base de quitosana com óleo essencial de Perilla frutescens, onde obtiveram resultados entre 9,365 a 22,344 N. Já Rocha et al. (2014) [1], obtiveram força de ruptura de biofilmes, apresentando variações entre 0,08 a 2,78 N. De acordo com Silva et al. (2019) [14], a resistência do biofilme à perfuração está correlacionada com o estado de compactação estrutural na rede da matriz polimérica, proposta para cada biopolímero.

A PVA do biofilme foi de $0,225 \mathrm{~g} \mathrm{~mm} \mathrm{~h}^{-1} \mathrm{~m}^{-2} \mathrm{kPa}$. De acordo com Rocha et al. (2014) [1], o alto valor de permeabilidade ao vapor de água para o biofilme de $C$. lanatus foi influenciado pelo glicerol, os autores complementam que quanto maior o teor deste plastificante, maior será a PVA. Silva et al. (2019) [14], avaliaram biofilmes a partir de fécula de mandioca com cafeína irradiada onde obtiveram PVA entre 0,3934-0,4638 $\mathrm{g} \mathrm{mm} \mathrm{h}^{-1} \mathrm{~m}^{2} \mathrm{kPa}$. Já Zhang et al. (2018) [9], avaliaram 
biofilmes a base de quitosana incorporados com óleo essencial de $P$. frutescens, onde obtiveram resultados médios de vapor de água entre 0,213 a $0,267 \mathrm{~g} \mathrm{~mm} \mathrm{~h}^{-1} \mathrm{~m}^{-2} \mathrm{kPa}$.

Na Tabela 2, estão apresentados os resultados para os parâmetros colorimétricos do filme convencional, e do biofilme de $C$. lanatus. A taxa de brilho $\mathrm{L}^{*}$ foi menor para o biofilme, com $\mathrm{L}^{*}$ de 26,25, e para o filme convencional com L* de 28,27. Silva et al. (2019) [14], obtiveram taxa de luminosidade com L* entre 46,78 e 59,65 para biofilmes a base de fécula de mandioca com cafeína irradiada.

Tabela 2: Parâmetros colorimétricos dos filmes, convencional (PVC) e biofilme de melancia.

\begin{tabular}{ccc}
\hline Parâmetros & Filme (PVC) & Biofilme \\
\hline $\mathbf{L}^{*}$ & $28,27 \pm 0,41$ & $26,25 \pm 0,07$ \\
$\mathbf{a}^{*}$ & $0,02 \pm 0,01$ & $1,17 \pm 0,04$ \\
$\mathbf{b}^{*}$ & $-0,81 \pm 0,07$ & $8,47 \pm 0,02$ \\
$\boldsymbol{C}^{*}$ & $0,81 \pm 0,07$ & $8,56 \pm 0,03$ \\
$\boldsymbol{h}^{\circ}$ & $271,43 \pm 0,65$ & $82,1 \pm 0,22$ \\
\hline
\end{tabular}

Médias em triplica seguida de ( \pm ) desvio padrão. $\mathrm{L}^{*}$ luminosidade ou brilho, $\mathrm{a}^{*}$ cromaticidade verde a vermelha, $b^{*}$ cromaticidade azul a amarelo, $\mathrm{C}^{*}$ croma e $\mathrm{h}^{\circ}$ ângulo de tonalidade.

A cromaticidade a* foi de 1,17 para o biofilme e de 0,02 para o filme convencional sintético, Tabela 2. Ambos tendendo para cor vermelha. Silva et al. (2019) [14], obtiveram biofilmes com tonalidade tendendo ao verde entre $a^{*}-0,75$ a $-0,86$, para biofilmes de fécula de mandioca incorporado com cafeína irradiada. Já para a cromaticidade $b^{*} \mathrm{o}$ biofilme apresentou resultado igual a 8,47 tendendo ao amarelo e de -0,81 para o filme convencional apresentando tonalidade para cor azul, Tabela 2. Silva et al. (2019) [14], encontraram cromas b* tendendo ao amarelo entre $-4,25$ a $-5,19$ em biofilmes de fécula de mandioca com cafeína irradiada. O Chroma $C^{*}$ foi de 8,56 para o biofilme e de 0,81 para o filme convencional, Tabela 2. Já o Hue foi de $h^{\circ}$ igual a 82,1 para o biofilme e de 271,43 para o filme convencional, Tabela 2. De acordo com Rocha et al. (2014) [1], a cor do biofilme pode influenciar tanto negativamente quanto positivamente na aceitação em aplicações comestíveis como não comestíveis (embalagens).

$\mathrm{Na}$ Figura 1, podem ser observados micrografias do filme convencional, e biofilme de C. lanatus, bem como, a modelagem matemática da área superficial.

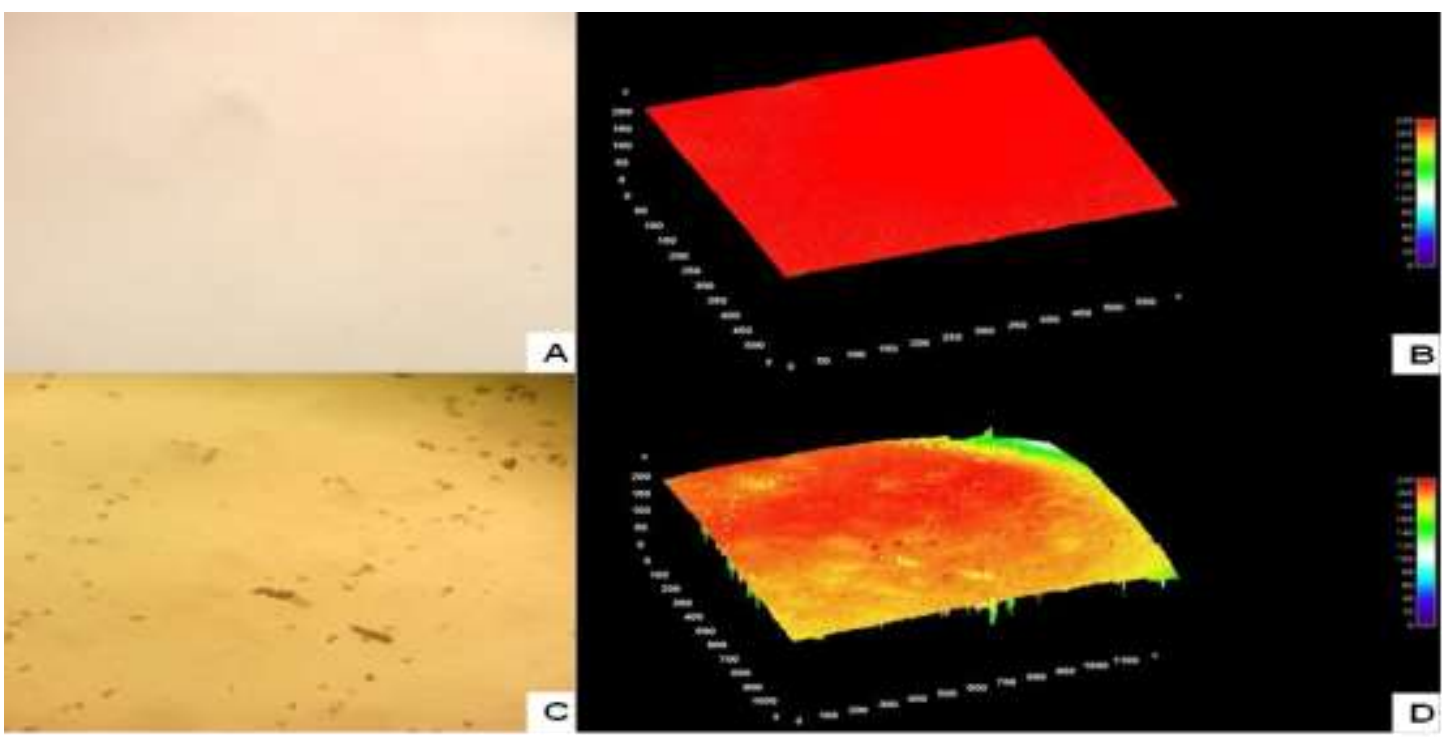

Figura 1: Fotomicrografias ópticas dos filmes convencional (PVC) em $(\boldsymbol{A})$ e biofilme em $(\boldsymbol{C})$, e modelagem matemática em $3 D$ da topografia da área superficial de ambos os filmes em $(\boldsymbol{B})$ e $(\boldsymbol{D})$, respectivamente. Imagens em $200 \mu \mathrm{m}$.

Na Figura 1, pranchas (A e C) estão apresentadas micrografias ópticas do filme convencional e biofilme de melancia. Em (A) pode ser observado homogeneidade da área superficial do filme $(\mathrm{PVC})$, e em (C) pequenos cristais formados durante o processo de convecção para o biofilme. $\mathrm{O}$ 
biofilme apresentou coloração amarelada, eficiente maleabilidade e aroma do fruto de $C$. lanatus. Brito et al. (2019) [19], avaliaram biofilmes de farinhas e pectina dos resíduos de laranja, maracujá e melancia, onde encontraram resultados próximos ao deste estudo, sem rugosidade, com aparência brilhante, eficiente maleabilidade, aroma frutal e coloração amarelada.

No modelo matemático em 3D, pode ser observado à topografia da área superficial em ambos os materiais. Ainda na Figura 1, pranchas (B e D) é possível observar a homogeneidade na interação intermolecular dos compostos que formam os diferentes grupos químicos em ambos os filmes comercial e biodegradável. Na prancha (B) o filme de PVC apresenta uma área plana se imperfeições, e já na prancha (D) são observadas cristais que tornam a área superficial do filme levemente ondulada. $\mathrm{O}$ uso de artifícios matemáticos empregados nas modelagens em imagens microscópicas, apresentaram neste estudo, bons resultados podendo ser empregados em outros estudos em conjunto para análise exploratória e na avaliação da topografia de biofilmes simples e compostos (incorporados ou em blendas), bem como compará-los com o filme convencional de PVC.

Na Figura 2, estão apresentadas micrografias por microscopia eletrônica de varredura (MEV) da área superficial em ambas as faces do biofilme de C. lanatus.

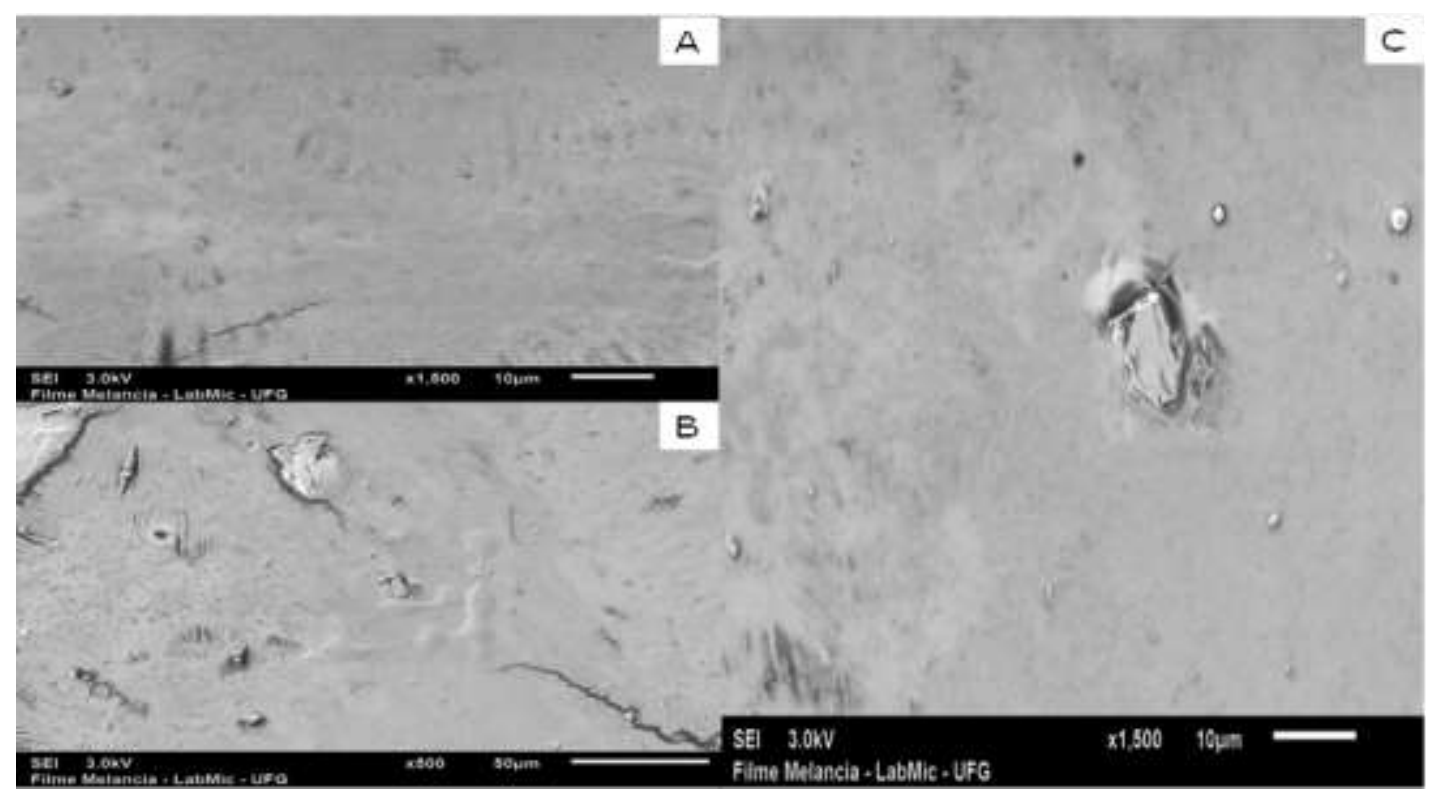

Figura 2: Eletromicrografias de varredura da área superficial em ambas as faces do biofilme de melancia (C. lanatus). Em (A) $10 \mu \mathrm{m},(B) 50 \mu \mathrm{m}$ e (C) $10 \mu \mathrm{m}$.

A análise por MEV do biofilme apresentou área superficial sem rachaduras Figura 2, prancha (A). Isso se deve as duas camadas de solução filmogênica utilizada para a confecção do biofilme, e também pela influência do glicerol como meio plastificante. O biofilme apresentou bons resultados de plasticidade. Esta característica está diretamente influenciada na troca de vapores entre o meio interno e externo, por exemplo, em uma embalagem, apresentando função que garanta maior vida de prateleira de um produto durante o armazenamento.

De acordo com Mali et al. (2010) [24], a ausência de rachaduras ou cavidades no biofilme, o meio, o fluxo de gases e também o vapor que permeia através da difusão molecular, é compreendido pela abertura do espaço vazio entre os segmentos de cadeias dos polímeros. Isto é ocasionado pelas oscilações dos segmentos, consecutivamente pelo deslocamento do permeante dentro do espaço vazio. Estudos realizados com quitosana também apresentam bons resultados com uma área superficial homogênea e sem rachaduras, exceto quando se incorpora óleos essenciais que tornam a superfície irregular e muitas das vezes quebradiça como observado por Zhang et al. (2018) [9].

Em contra partida, a alta temperatura e o fluxo de ar forçado durante o processo de secagem, contrastou na microestrutura do biofilme de C. lanatus, onde apresentaram formação de cristais e manchas de coloração escura como observado na Figura 2, pranchas (B e C). O uso de substâncias para incorporação em soluções filmogênicas demonstram causar deformidades nos biofilmes, como 
observados por Zhang et al. (2018) [9], e Marin et al. (2013) [25], onde apresentaram em seus estudos deformidades e rugosidades nos filmes incorporados com óleos essenciais e aldeído fenólico (vanilina).

Na Figura 3, estão apresentados os espectros de absorção na região do infravermelho médio com transformada de Fourier (FT-IR), para o filme comercial convencional e do biofilme de C. lanatus.

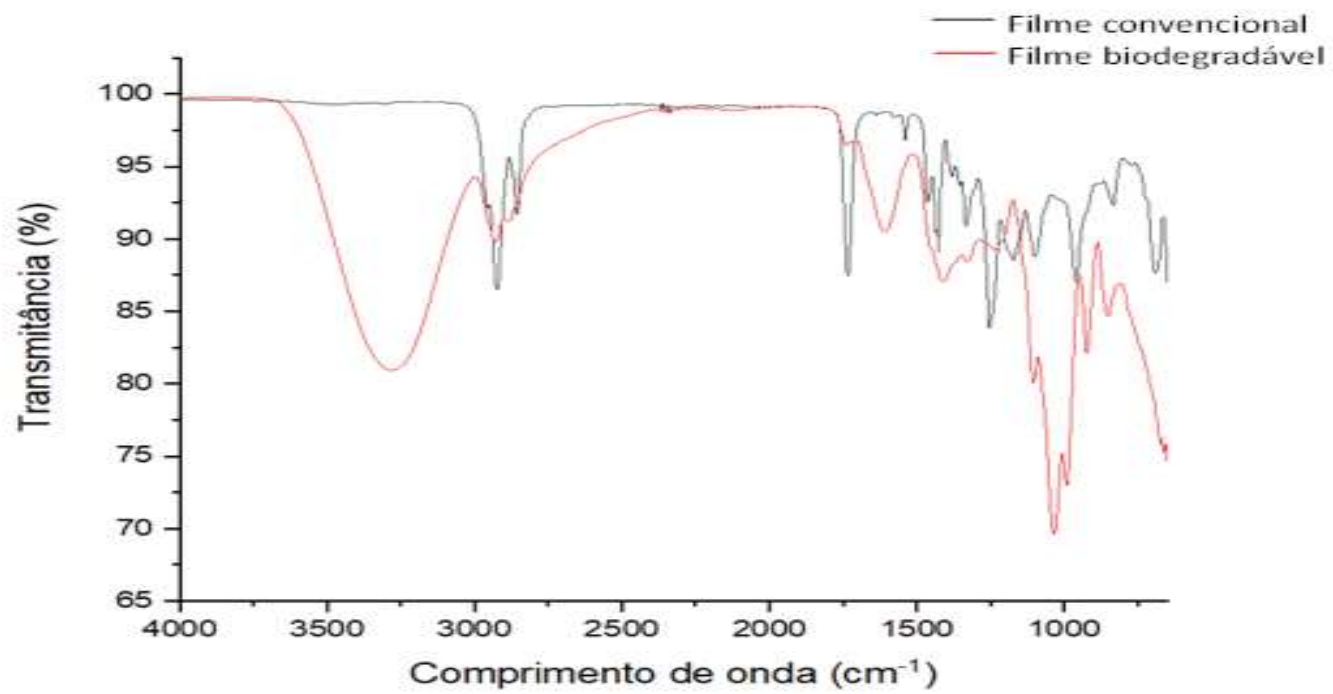

Figura 3: Espectros de absorção na região média do infravermelho entre $4000-650 \mathrm{~cm}^{-1}$ do filme convencional $(P V C)$ e biofilme de Citrullus lanatus.

Os espectros por infravermelho em ambos os filmes de PVC e biodegradável foram comparados conforme Figura 3, para demonstrarem as diferenças entre os tipos de grupamentos químicos presentes em cada polímero. Em especial, o biofilme apresentou maior quantidade de grupos funcionais, quando comparado ao filme de PVC. Esta diferença está relacionada devido à matriz natural apresentar maior quantidade de compostos químicos com diferentes grupos funcionais.

$\mathrm{O}$ biofilme apresentou uma banda vibracional longa em $3272 \mathrm{~cm}^{-1}$ pertencente ao grupo $\mathrm{O}-\mathrm{H}$, devido à higroscopicidade da matriz polimérica. Já Mei e Oliveira (2017) [26], observaram uma banda entre 3300-3500 cm $\mathrm{cm}^{-1}$, referente à vibração axial característica do grupo O-H. Uma banda em $2931 \mathrm{~cm}^{-1}$ é atribuída à vibração do alongamento do grupamento funcional C-H. O mesmo foi observado por Zhang et al. (2018) [9], em biofilmes de quitosana incorporado com óleo esencial, onde apresentaram uma banda em $2873 \mathrm{~cm}^{-1}$.

Ainda na Figura 3, bandas no comprimento de ondas em 1595; 1412 e $1101 \mathrm{~cm}^{-1}$ podem corresponder às vibrações do estiramento do grupo $\mathrm{C}=\mathrm{O}$, vibrações de flexão dos grupos $\mathrm{N}-\mathrm{H}, \mathrm{C}$ $\mathrm{N}$, alongamento de $\mathrm{N}-\mathrm{H}$ e ou vibrações do estiramento da banda $\mathrm{C}-\mathrm{O}-\mathrm{C}$ respectivamente. No comprimento de ondas em $1033 \mathrm{~cm}^{-1}$ corresponde ao alongamento vibracional do grupo C-O, e em $848 \mathrm{~cm}^{-1}$ apresenta uma banda correspondente à vibração de flexão do grupo $\mathrm{O}-\mathrm{H}$. Resultados próximos ao deste estudo, foi observado por Jahed et al. (2017) [8], com biofilmes de quitosana incorporados a celulose, ligninocelulose e óleo essencial de Ocimum vulgare.

O filme convencional de PVC apresenta diferenças entre a estrutura e a matéria-prima polimérica advinda de produtos do petróleo. Na Figura 3, pode ser observada bandas em 2923 e $1463 \mathrm{~cm}^{-1}$ que são atribuídas a vibrações angulares do grupo $\mathrm{H}-\mathrm{C}-\mathrm{H}$ no plano, e aos estiramentos do grupo $\mathrm{C}-\mathrm{H}$ alquilas. Em $2855 \mathrm{~cm}^{-1}$ apresenta banda correspondente à deformação axial do grupo C-H de alcanos [27]. Uma banda é atribuída ao estiramento da carbonila em $1733 \mathrm{~cm}^{-1}$. Souza et al. (2009) [28], avaliaram filmes plásticos onde obtiveram essa banda característica do estiramento da carbonila em $1730 \mathrm{~cm}^{-1}$. Uma banda em $1253 \mathrm{~cm}^{-1}$ corresponde à deformação do grupo C-H no plano do anel, e a banda em $692 \mathrm{~cm}^{-1}$ é atribuída à vibração do grupo C-Cl. O filme plástico de PVC é considerado um polímero atático com tendência sindiotática [29]. 


\section{CONCLUSÃO}

O biofilme de Citrullus lanatus apresentou homogeneidade, fácil manipulação e desprendimento do suporte de secagem, apresentando importantes características físico-químicas, mecânicas, excepcionais atividades antioxidantes, bem como compostos fenólicos totais e estruturais promovidos pelo biopolímero. O biofilme apresentou coloração amarelada, não sendo uma boa opção para uso em geral como embalagem para alimentos. Entretanto, a sua aplicabilidade pode ser utilizada na produção de sacolas plásticas biodegradáveis e para revestimento em produtos alimentícios especiais como, salames e linguiças defumadas garantindo proteção contra choques mecânicos, baixa PVA, baixa solubilidade e alta integridade do biofilme garantindo a qualidade dos produtos, bem como, durante o manuseio e manipulação atendendo ao seu uso como embalagem biodegradável.

\section{AGRADECIMENTOS}

Ao Instituto Federal de Educação, Ciência e Tecnologia Goiano, Campus Rio Verde; a Universidade Federal de Goiás; a Universidade Estadual Júlio de Mesquita Filho, Campus Araraquara; aos laboratórios de Química Tecnológica, Pós-Colheita, Produtos Naturais, Laboratório multiusuário de microscopia de alta resolução; a Central Analítica Multiusuário; aos órgãos de fomento em pesquisa CNPq, CAPES, FINEP e FAPEG, esta última, pela bolsa de mestrado em Agroquímica para o primeiro autor.

\section{REFERÊNCIAS BIBLIOGRÁFICAS}

1. Rocha GO, Farias MG, Carvalho CWP, Ascheri JLR, Galdeano MC. Filmes compostos biodegradáveis a base de amido de mandioca e proteína de soja. Polímeros. 2014;24(5):587-595, doi:10.1590/01041428.1355

2. Henriques CM, Cereda, MP, Sarmento SBS. Características físicas de filmes biodegradáveis produzidos a partir de amidos modificados de mandioca. Ciênc Tecnol Aliment. 20082;8(1):231-40.

3. Tang R, Du Y, Zheng H, Fan L. Preparation and characterization of soy protein isolate-carboxymethylated konjac glucomannan blend films. J Appl Poly Sci. 2003;88(5):1095-99, doi:10.1002/app.11703

4. Oliveira JB, Grangeiro LC, Sobrinho JE, Moura MSB, Carvalho CAC. Rendimento e qualidade de frutos de melancias em diferentes épocas de plantio. Rev Caatinga. 2015;28(2):19-25.

5. Souza CO, Silva LT, Druzian JI. Estudo comparativo da caracterização de filmes biodegradáveis de amido de mandioca contendo polpas de manga e de acerola. Química Nova. 2012;35(2):262-67.

6. IAL - Instituto Adolfo Lutz. Métodos físico-químicos para análise de alimentos. $4^{\mathrm{a}}$ Ed., $1^{\mathrm{a}}$ Ed. Digital, 2008. $1020 \mathrm{p}$.

7. Abdollahi M, Rezaei M, Farzi G. Improvement of active chitosan film properties with rosemary essential oil for food packaging. Int J Food Sci Tech. 2012;47:847-53, doi: 10.1111/j.1365-2621.2011.02917.x

8. Jahed E, Khaledabad A, Bari MR, Almasi H. Effect of cellulose and lignocellulose nanofibers on the properties of Origanum vulgare ssp. gracile essential oil-loaded chitosan films. React Func Poly. 2017;117:70-80, doi:10.1016/j.reactfunctpolym.2017.06.008.

9. Zhang Z-J, Li N, Li H-Z, Li X-J, Cao J-M, Zhang G-P, He D-L. Preparation and characterization of biocomposite chitosan film containing Perilla frutescens (L.) Britt. essential oil. Ind Crops Prodets. 2018;112:660-7, doi:10.1016/j.indcrp.2017.12.073.

10. Menezes Filho ACP, Silva MA, Pereira AV, Oliveira Filho JG, Castro CFS. Parâmetros físico-químicos, tecnológicas, atividade antioxidante, conteúdo de fenólicos totais e carotenóides das farinhas dos frutos do jatobá-do-cerrado (Hymenaea stigonocarpa Mart. Ex Hayne). Multi-Science J. 2019;1(12):1-4.

11. Martucci JF, Ruseckaite RA. Tensile properties, barrier properties, and biodegradation in soil of compression - moldel gelatine-dialdehyde starch films. J Appl Poly Sci. 2009;112: 2166-78, doi:10.1002/app.29695.

12. Infante J, Selani MM, Toledo NMV, Silveira-Diniz MF, Alencar SM, Spoto MHF. Atividade antioxidante de resíduos agroindustriais de frutas tropicalis. Rev Ali \& Nutri. 2013;24(1): 87-91.

13. Menezes Filho ACP, Oliveira Filho JG, Christofoli M, Castro CFS. Atividade antioxidante e compostos bioativos em especies de um fragment de Cerrado Goiano tipo cerradão. Colloq Agrariae. 2019;15(1): 18, doi:10.5747/ca.2019.v15, n1.a264.

14. Silva MCS, Lima PKD, Silveira MFA, Arthur V, Souza ARM. Avaliação das propriedades físicoquímicas e mecânicas de filmes de fécula de mandioca incorporado com cafeína irradiada. Rev Desafios. 2019;6: 91-99, doi:10.20873/uft.2359365220196Especialp91. 
15. Cleirici MTPS, Kallmann C, Gaspi FOG, Morgano MA, Martínez-Bustos F, Chang YK. Physical, chemical and technological characteristics of Solanum lycocarpum A. St. - Hill (Solanaceae) fruit flour and starch. Food Res Int. 2011;44: 2143-50, doi:10.1016/j.foores.2011.01.060

16. Costa DMA, Santos AF, Silva ED, Silva IA. Desenvolvimento e Caracterização de filmes à base de amido de feijão macáçar (Vigna unguiculata (L.) Wap). Holos. 2017;7:2-16, doi:10.15628/holos.2017.6318

17. Dantas EA, Costa SS, Cruz LS, Bramont WB, Costa AS, Padilha FF, Druzian JI, Machado BAS. Caracterização e avaliação das Propriedades antioxidantes de filmes biodegradáveis incorporados com polpas de frutas tropicalis. Ci Rural. 2015;45(1):142-8, doi:10.1590/0103-8478cr20131458

18. Carissimi M, Simone HF, Rech R. Effect of microalgae addition on active biodegradable starch film. Algal Res. 2018;32:201-9, doi:10.1016/j.algal.2018.04.001

19. Britto TB, Carrajola JF, Gonçalves ECBA, Martelli-Tosi M, Ferreira MSL. Fruit and vegetable residues flours with different granulometry range as raw material for pectin-enriched biodegradable film preparation. Food Res Int. 2019;121:412-21, doi:10.1016/j.foodres.2019.03.058

20. Santana MCCB, Machado BAS, Silva TN, Nunes IL, Druzian JI. Incorporção de urucum como aditivo antioxidante em embalagens biodegradáveis a base de quitosana. Ci Rural. 2013;43(3):544-50.

21. Campagner MR, Moris VAS, Pitombo LM, Carmo JB, Paiva JMF. Filmes poliméricos baseados em amido e lignossulfonatos: preparação, Propriedades e avaliação da biodegradação. Polímeros. 2014;24(6):740-51, doi:10.1590/0104-1428.1700

22. Kim S, Baek S-K, Song KB. Physical and antioxidant properties of alginate films prepared from Sargassum fulvellum with black chokeberry extract. Food Pack S Life. 2018;18:157-63, doi:10.1016/j.fpsl.2018.11.008

23. Mir SA, Dar BN, Wani AA, Shah MA. Effect of plant extracts on the techno-functional properties of biodegradable packaging films. Trends F Sci Tech. 2018;80:141-54, doi:10.1016/j.tifs.2018.08.004

24. Mali S, Grossmann MVE, Yamashita F. Filmes de amido: produção, Propriedades e potencial de utilização. Semina: Cien Agrárias. 2010;31(1):137-56.

25. Marin L, Stoica I, Mare M, Dinu V, Simionescu BC, Barboiu M. Antifugal vanillin-imino-chitosan biodynameric films. J Mat Chemistry. 2013;1(27):3353-8, doi:10.1039/C3TB20558D

26. Mei LH, Oliveira N. Caracterização de um compósito polimérico biodegradável utilizando poli ( $\varepsilon$ caprolactona) e borra de café. Polímeros. 2017;27:99-109, doi:10.1590/0104-1428.2139

27. Silverstein RM, Webster FX, Kiemle DJ. Identificação espectrofotométrica de compostos orgânicos. 2013. $7^{\mathrm{a}}$ Ed., LTC, 490 p.

28. Souza ML, Corio P, Temperini MLA, Temperini JA. Aplicação de espectroscopia Raman e infravermelho na identificação e quantificação de plastificantes em filmes comerciais de PVC esticável. Química Nova. 2009;32(6):1452-6.

29. Gonzalez N, Mugica A, Fernandez-Berridi MJ. Application of high resolution thermogravimetry to the studey of thermal stability of poly(vinyl chloride) resins. Poly Degrad Stability. 2006;91:629-33, doi:10.1016/j.polymdegradstab.2004.12.015 